\title{
Numerical Study of a Fragile Three-Dimensional Kinetically Constrained Model
}

\author{
Ludovic Berthier ${ }^{\dagger}$ and Juan P. Garrahan*," \\ Laboratoire des Verres UMR 5587, Université Montpellier II and CNRS, 34095 Montpellier, France, and \\ School of Physics and Astronomy, University of Nottingham, Nottingham, NG7 2RD United Kingdom
}

Received: October 4, 2004; In Final Form: December 15, 2004

\begin{abstract}
We numerically study the three-dimensional generalization of the kinetically constrained east model, the north-or-east-or-front (NEF) model. We characterize the equilibrium behavior of the NEF model in detail, measuring the temperature dependence of several quantities: $\alpha$-relaxation time, distributions of relaxation times, dynamic susceptibility, dynamic correlation length, and four-point susceptibility. We show that the NEF model describes quantitatively experimental observations over an exceptionally wide range of time scales. We illustrate this by fitting experimental data obtained both in the mildly supercooled regime by optical Kerr effect and close to the glass transition by dielectric spectroscopy.
\end{abstract}

\section{Introduction}

Supercooled liquids can be studied using theoretical models where the density field dynamics is replaced by a coarse-grained mobility field evolving with simple empirical rules based on the idea of dynamic facilitation. ${ }^{1}$ Kinetically constrained models (KCM), such as the Fredrickson-Andersen (FA) model $^{2}$ and the east model, ${ }^{3,4}$ have been shown to reproduce well the phenomenology of supercooled liquids. An important feature of these models is that their dynamics becomes spatially correlated;, that is, growing time scales are accompanied by growing dynamical lengthscales, giving rise to dynamic heterogeneity as observed in experiments and simulations. ${ }^{7-10}$

In this paper, we study by means of extensive numerical simulations the three-dimensional generalization of the east model, ${ }^{3}$ the north-or-east-or-front (NEF) model. ${ }^{11}$ This model is a realization of the "arrow" model of ref 12 but with an externally imposed directional preference, whereas in the arrow model, excitations carry an orientation which locally determines the directionality of the dynamics, making the system isotropic. ${ }^{12}$ These models were shown to reproduce the dynamic behavior of a wide range of fragile materials. ${ }^{12}$ The present study is complementary to that of refs 13 and 14 that considered systems with fully isotropic dynamic facilitation, such as the threedimensional FA model, which describes strong materials.

The principal aims of this work are the following:

(i) Quantitative Characterization of the Fragile Limit. We report numerical results for a wide range of dynamic observables in the NEF model, describing in detail distributions of the relevant time scales and lengthscales. We also confirm some of the predictions for the arrow model of ref 12 .

(ii) Comparison to the Strong Limit. We contrast our results for the NEF model to those obtained in refs 13 and 14 which dealt with the strong limit of isotropic facilitation, such as the three-dimensional FA model.

(iii) Comparison to Experimental Data. We use the NEF model results to fit experimental data over a wide range of relaxation times, covering both the onset of supercooling and the regime close to the glass transition.

\footnotetext{
* To whom correspondence should be addressed.

† Université Montpellier II and CNRS.

$\doteqdot$ University of Nottingham.
}

The paper is organized as follows. We first describe the model and some technical details in section II. We then turn to the study of the relevant time scales and how they are distributed and evolve with temperature in section III. We study spatial aspects of the dynamic in section IV: dynamic heterogeneity and four-point correlations. We compare our numerical results to experimental data in section $\mathrm{V}$ and conclude the paper in section VI.

\section{Model and Simulation Details}

The NEF model is defined by the Hamiltonian

$$
H=\sum_{i=1}^{N} n_{i}
$$

where $n_{i}=0,1$ are $N=L^{3}$ binary variables defined on each site of a cubic lattice of linear size $L$, which has periodic boundary conditions. Physically, $n_{i}=1\left(n_{i}=0\right)$ describes at a coarse-grained level a site which is mobile (immobile).

The dynamics of the model can be written as follows:

$$
n_{i}=0 \underset{\sigma_{i}(1-c)}{\stackrel{\sigma_{i} c}{\rightleftarrows}} n_{i}=1
$$

where

$$
c=\left\langle n_{i}\right\rangle=\frac{1}{1+\exp (1 / T)}
$$

is the mean concentration of mobile regions and $O_{i}$ is the kinetic constraint

$$
O_{i}=1-\prod_{\langle j, i\rangle}\left(1-n_{j}\right)
$$

The prime indicates that the product is restricted to those three sites, $j$, which are north, front, and east nearest neighbors of site $i$. The kinetic constraint (4) implies that site $i$ cannot change state unless at least one of those three neighbors is in the state $n_{j}=1$. The system is therefore more constrained than the corresponding one-spin facilitated FA model, where any of its six neighbors can facilitate the site $i$. In the arrow model, 


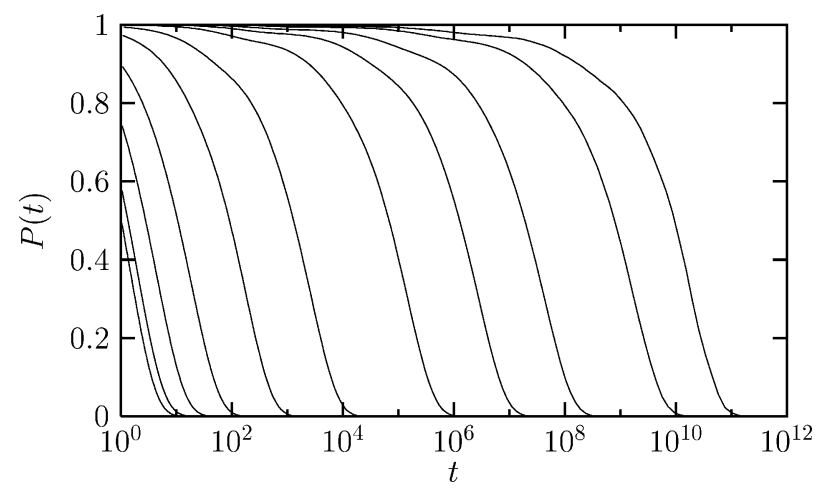

Figure 1. Persistence function, eq 5 , for temperatures decreasing from left to right: $T=4.0,2.0,1.0,0.6,0.4,0.3,0.23,0.2,0.18,0.16$, and 0.15 .

anisotropy is only local and results physically from the empirical observation that facilitation has locally a preferred direction that may persist over a certain length scale even though the system is on average totally isotropic. ${ }^{12}$ The NEF model corresponds therefore to having an infinitely large persistence length of dynamic facilitation. This is physically unrealistic but constitutes a useful limit effectively describing systems with a large but finite persistence of dynamic facilitation. ${ }^{15}$

We performed Monte Carlo simulations of the NEF model for several temperatures in the range $T \in[0.15,5.0]$, covering over 11 orders of magnitude in relaxation times. Such large time scales can be simulated using a continuous time algorithm. ${ }^{17}$ As we shall see, this dynamical slow-down is accompanied by the growth of dynamic spatial correlations, and one has to pay attention to possible finite size effects. ${ }^{18}$ This is however not a serious problem in the present model because even at very large time scales spatial correlations are not very large (see below). At the lowest temperature simulated a system size of $N=32^{3}$ proved to be large enough. Compare this to the system size $N=160^{3}$ used in the strong limit to simulate similar time scales. ${ }^{14}$ Equilibrium behavior is trivial due to the noninteracting Hamiltonian (1). It is straightforward to produce independent equilibrated initial configurations. Simulations therefore only consist of production runs. Averages are then performed over truly independent initial configurations.

\section{Time Scales}

A. Global Dynamics. We first consider the spatially averaged dynamics, which may be probed via the mean persistence function

$$
P(t)=\left\langle\frac{1}{N_{i}} \sum_{i=1}^{N} P_{i}(t)\right\rangle
$$

where $P_{i}(t)$ is the single-site persistence function at time $t$, which takes the value 1 if site $i$ has not flipped up to time $t$, and the value 0 otherwise. As discussed in ref 19, the persistence function $P(t)$ can be seen as the analogue of the self-intermediate scattering function, $F_{\mathrm{s}}(k, t)$, of a supercooled liquid at wavelengths comparable to the particle diameter. ${ }^{20}$ Figure 1 shows, as expected, that the dynamics slows down markedly when temperature is decreased below $T_{0} \approx 1.0$, indicating the onset of slow dynamics in this model. ${ }^{21,22}$ Physically, $T_{0}$ corresponds to the energy scale of the problem, i.e., the energy needed to create an excitation, see eq 3 .

The shape of the mean persistence function will be discussed below in some detail. For the moment, we simply note that the long time decay is nonexponential, as is commonly observed

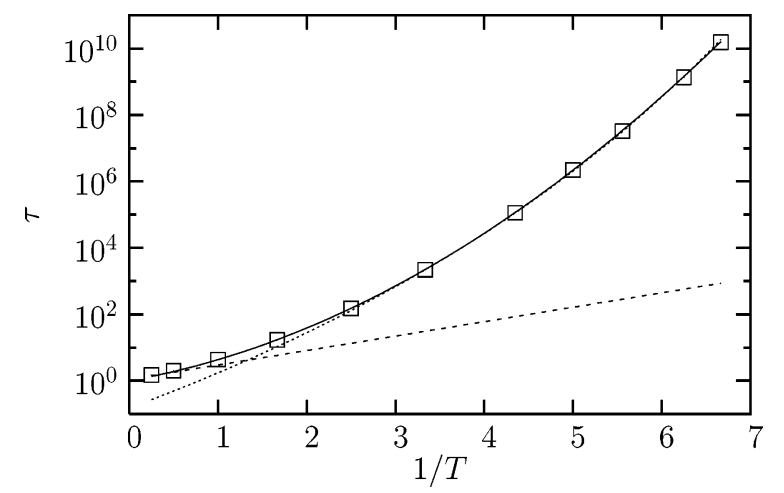

Figure 2. Points: Mean relaxation time from $P(\tau)=\mathrm{e}^{-1}$. The lines represent various fits. Dashed line is the high-temperature Arrhenius behavior, $\tau \sim \exp (1 / T)$. Full line is $\tau(T) \sim \exp (\Delta(T) / T)$ with $\Delta(T)=$ $0.634 / T+1.1$. The dotted line shows that a Vogel-Fulcher form, $\tau \sim$ $\exp [2.3 /(T-0.06)]$, fits the low-temperature data with an unphysical finite $T$ singularity.

in supercooled liquids. Moreover, the short-time dynamics also presents nontrivial features, see for instance the curve for $T=$ 0.15 in Figure 1.

The mean relaxation time, $\tau(T)$, is extracted via the usual relation $P(\tau)=\mathrm{e}^{-1}$. In the present context, $\tau$ represents also the $\alpha$ relaxation of the model. The temperature dependence of $\tau$ is shown in Figure 2. The main observation from this figure is that the mean relaxation time grows with decreasing temperature in a super-Arrhenius manner, see Figure 2. The NEF model behaves as a fragile supercooled liquid, as expected. ${ }^{12}$ Note however that the ratio $T_{0} / T_{\mathrm{g}} \approx 7$ that can be extracted from Figure 2 is about three times smaller in fragile liquids such as $o$-terphenyl.

Various fits are also included in Figure 2. The high temperature behavior is well described by a naive mean-field approximation $^{21,23}$

$$
\tau_{\mathrm{MF}} \sim c^{-1}
$$

This behavior breaks down below $T_{0}$, where dynamics is dominated by local fluctuations of the mobility. Generalizing to three dimensions the results for the east model, $3,12,24$ we expect that in the limit of small temperature, the time scale behaves as

$$
\tau \sim c^{-\Delta(T)}
$$

with $\Delta(T) \approx b / T$ at low $T$, where $b$ is a constant. Since $c \sim$ $\mathrm{e}^{-1 / T}$, eq 7 implies an exponential inverse temperature squared dependence at low temperatures, $\tau \sim \mathrm{e}^{b / T^{2}}$. In fact, an empirical fit using

$$
\Delta(T)=a+\frac{b}{T}
$$

allows one to describe the whole temperature range studied, using $b=0.634$ and $a=1.1$. The supposedly exact lowtemperature result, $\Delta(T)=(3 \ln 2 T)^{-1}$, works well for very large relaxation times. Indeed, we find that a plot of $T^{2} \log \tau$ vs $1 / T$ nicely converges to the theoretical value $(3 \ln 2)^{-1}$ (not shown). At low temperatures, the $\alpha$ relaxation in the NEF model therefore follows a Bässler law. ${ }^{25}$

Figure 2 also shows that the low $T$ data can be fit using the Vogel-Fulcher law, $\tau_{\mathrm{VF}} \sim \exp \left[A /\left(T-T_{0}\right)\right]$. The Bässler and Vogel-Fulcher curves are hardly distinguishable for over 8 orders of magnitude, as has been observed before when fitting 


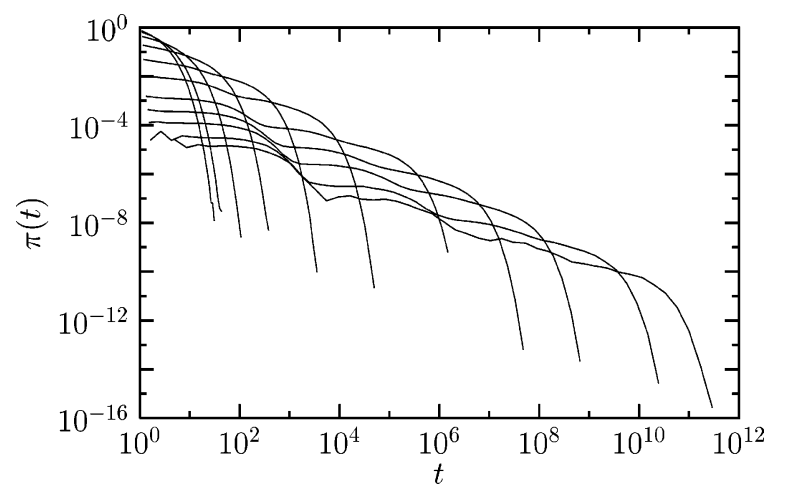

Figure 3. Distribution of persistence time $\pi(t)$, see eq 9 , for temperatures as in Figure 1, decreasing from left to right.

experimental data. ${ }^{25}$ In our case, it is evident that the finite temperature singularity of the Vogel-Fulcher law is completely unphysical.

As will be discussed below, the fragile behavior of the NEF model results from the existence of a hierarchy of lengthscales governing the dynamical behavior. It is therefore not surprising that the relaxation time in this model does not follow the AdamGibbs relation which is argued to link dynamics and thermodynamics of supercooled liquids. ${ }^{26,27}$ For the NEF model, the entropy has essentially an Arrhenius behavior at low temperatures. The Adam-Gibbs relation thus predicts the relaxation time to grow as a double exponential of the inverse temperature. This vastly overestimates the growth of $\tau$, which follows the Bässler law, eqs 7 and 8. In the same vein, naively extracting a Kauzmann temperature by linearly extrapolating the entropy to zero in the same temperature range where the Vogel-Fulcher law seems to apply (see Figure 2 ) gives a value $T_{\mathrm{K}} \approx 0.16$, which is very different from the Vogel-Fulcher temperature $T_{0} \approx 0.06$ found above. Although $T_{0}$ and $T_{\mathrm{K}}$ are ill-defined temperatures in our case, experimentally they are often found to be close in fragile liquids, a feature which is not quantitatively reproduced by the NEF model.

B. Distribution of Relaxation Times. The mean relaxation time $\tau(T)$ captures only in part the relaxation behavior of the model. We consider in this subsection the distribution of relaxation times, $\pi(t)$, related to the mean persistence function $\mathrm{via}^{21}$

$$
P(t)=\int_{t}^{\infty} \mathrm{d} t^{\prime} \pi\left(t^{\prime}\right)
$$

These distributions are shown in Figure 3.

As mentioned above, the long time tail of the persistence function is well-described by a stretched exponential form, which implies the following long time behavior for the distribution of relaxation times

$$
\pi(t) \sim \frac{t^{\beta-1}}{\tau^{\beta}} \exp \left[-\left(\frac{t}{\tau}\right)^{\beta}\right]
$$

valid for when $t \gg \tau$. As for the east model, ${ }^{4,28,29}$ we find that the stretching exponent $\beta$ decreases with $T$ in the regime $T<$ $T_{0}$, from its high-temperature value $\beta=1$.

As can be guessed from Figures 1 and 3, using $\tau(T)$ and $\beta$ $(T)$ as unique fitting parameters does not allow for a satisfactory description of the whole decay of the persistence function and distribution of relaxation times. This becomes evident when data are presented in an alternative way. Following the experimental literature, we show the data obtained from the distribution of time scales in a frequency representation via $^{30}$

$$
\chi^{\prime \prime}(\omega)=\operatorname{Im} \int_{-\infty}^{\infty} \pi(\log (\tau)) \frac{1}{1+i \omega \tau} \mathrm{d} \log \tau
$$

as is often done with dielectric susceptibility measurements. This will also allow us to make quantitative comparisons to experiments below. The results for $\chi^{\prime \prime}(\omega)$ are displayed in Figure 4. At temperature $T=0.18$, we show on top of the data the Fourier transform of the stretched exponential fit to the long time tail of $P(t)$. The fit is clearly incorrect in this representation, although it would look acceptable in most of a plot such as in Figure 1. In fact, deviations appear when $\omega \tau>1$, corresponding to $t<$ $\tau$ in the time domain. This reveals the presence of "additional processes" on the "high-frequency flank" of the $\alpha$ relaxation.

This feature is obviously reminiscent of the "high-frequency", or "Nagel", wing extensively studied by dielectric spectroscopy in several materials. ${ }^{31}$ The wing is usually observed in fragile glass formers, but its precise nature has not been fully elucidated. Despite initial claims of certain universal behavior of the highfrequency wing, more recent investigations seem to favor the interpretation that the phenomenon does not obey universal scaling. ${ }^{31}$ This is compounded by the observation that the amplitude of the phenomenon also seems to depend on the technique used to study it. ${ }^{32}$

In our case, the physical interpretation of the wing is very clear. The relaxation of the NEF model proceeds in a hierarchical manner, just as in the one-dimensional case. ${ }^{4,6}$ To relax a domain of immobile regions of size $l$, a region of size $\not 2$ must first be relaxed, which itself necessitates the relaxation of a domain of size 14 , etc., down to the smallest size $\ell=1$. This implies an energy cost $\Delta E(\zeta) \sim \ln /$, from which the Bässler form, eq 8, and stretched exponential decay, eq 10, follow. ${ }^{6}$ Interestingly, the hierarchy also shows up in the distributions of Figures 3 and 4 at time scales shorter than the $\alpha$ relaxation in a manner reminiscent of the experimental finding of additional short-time processes. Due to the underlying lattice structure of the model, the hierarchy is discrete rather than continuous, as can be indeed observed in Figure 4. Notice also that there is no 'fast' process at the microscopic time scale, $\omega \sim 1$, in our system. This is a consequence of coarse-graining by which molecular vibrations are removed.

These results show that the pattern of dynamical relaxation of the NEF model is quantitatively accurate on a wide frequency range. This will be shown explicitly in section $\mathrm{V}$ where dielectric susceptibility data taken close to the experimental glass transition are compared to the NEF model results. We recall that in the strong case the large-time decay was found to be purely exponential, whereas short-time processes had a much smaller magnitude. ${ }^{14}$ This prediction is however hard to confirm experimentally since strong liquids are not easy to study by dielectric spectroscopy. ${ }^{33}$

Physically, our results also suggest that the high-frequency wing observed in fragile glass-formers is an intrinsic feature of the $\alpha$ relaxation linked in a direct way to their dynamical hierarchical structure which is also at the origin of stretched relaxation and fragile behavior.

When the relaxation time is moderate, an even more complex behavior is observed due to the influence of clusters of defects. Such dynamic objects are irrelevant at low temperatures but can influence the short-time dynamics just below $T_{0}$, as discussed in detail in ref 21. These clusters were in particular shown to be responsible for the temperature behavior of a number of quantities discussed in some numerical works. Clusters also produce additional short-time processes visible in the distributions $\pi(t)$ when the temperature is not too low. ${ }^{21}$ Interestingly, in the present context, these patterns closely 


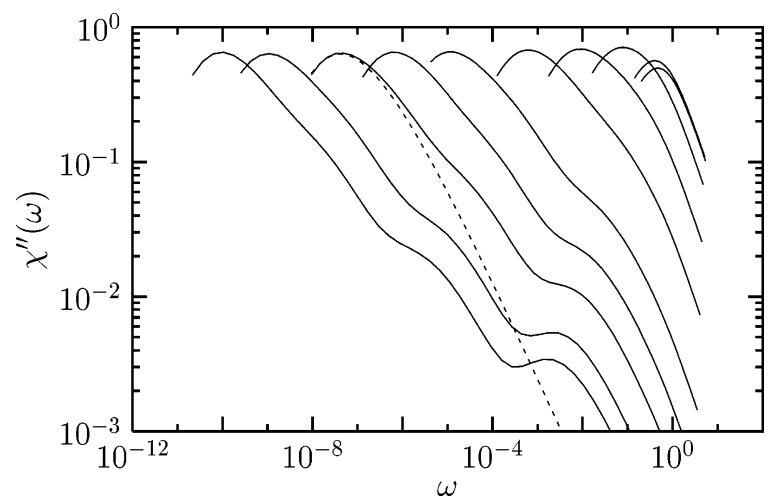

Figure 4. Imaginary part of the dynamic susceptibility defined in eq 11, for temperatures as in Figure 1, decreasing from right to left. For $T=0.18$, we also show as a dashed line the Fourier transform of the stretched exponential fit to the long time tail of the persistence function, revealing "additional processes" on the "high-frequency flank" of the $\alpha$ relaxation.

resemble the ones observed experimentally in mildly supercooled liquids and are usually explained in terms of modecoupling theory. ${ }^{34}$ This will be demonstrated in section $\mathrm{V}$, where data recently fitted via mode-coupling theory ${ }^{35}$ are also fitted using the NEF model.

\section{Length Scales}

A. Dynamic Heterogeneity. The growth of time scales in the NEF model is accompanied by growing spatial correlations as the system approaches its critical point at $T=0$. These correlations are purely dynamical in origin and give rise to dynamic heterogeneity. ${ }^{6,19}$ Figures 5 and 6 serve to illustrate this phenomenon, as we now explain.

We quantify the local dynamics via the local persistence function $P_{i}(t)$. For a given temperature, we run the dynamics for a time $t^{*}$, such that $P\left(t^{*}\right)=1 / 2$, meaning that half of the sites have flipped at least once. Persistent (immobile) spins are colored white, for which $P_{\mathrm{i}}\left(t^{*}\right)=1$, and transient (currently or previously mobile) spins are colored black, for which $P_{\mathrm{i}}\left(t^{*}\right)=$ 0 . Figure 5 shows the local persistence function for the NEF model at two different temperatures, $T_{0}=1.0$ and $T=0.15 \ll$ $T_{0}$. Clearly, the low-temperature dynamics is spatially heterogeneous, and the spatial correlations of the local dynamics grow as $T$ is decreased. The "critical" nature of dynamic clusters is apparent: the pictures are reminiscent of the spatial fluctuations of an order parameter close to a continuous phase transition, such as the magnetization of an Ising model near criticality. In our case, the order parameter is a dynamic object, and the persistence function and the critical fluctuations are purely dynamical in origin. ${ }^{18}$

It is interesting to note that these figures are qualitatively different from the ones obtained in the strong case where dynamic facilitation is isotropic. One can clearly distinguish in Figure 5 the north, east, and front directions of facilitation, implying that wandering of excitations in the other three directions is forbidden. Domains of Figure 5 appear much less rough than the ones obtained in the isotropic case. ${ }^{14}$ In that sense, increasing the fragility is similar to increasing the "surface tension" of the dynamic domains observed in Figure 5. The same observation applies to an even more fragile system, the two-spin facilitated FA model in two dimensions, where the corresponding domains resemble a polydisperse assembly of squares. ${ }^{5}$

The qualitative observations of dynamic heterogeneity performed in numerical or experimental works can also be made
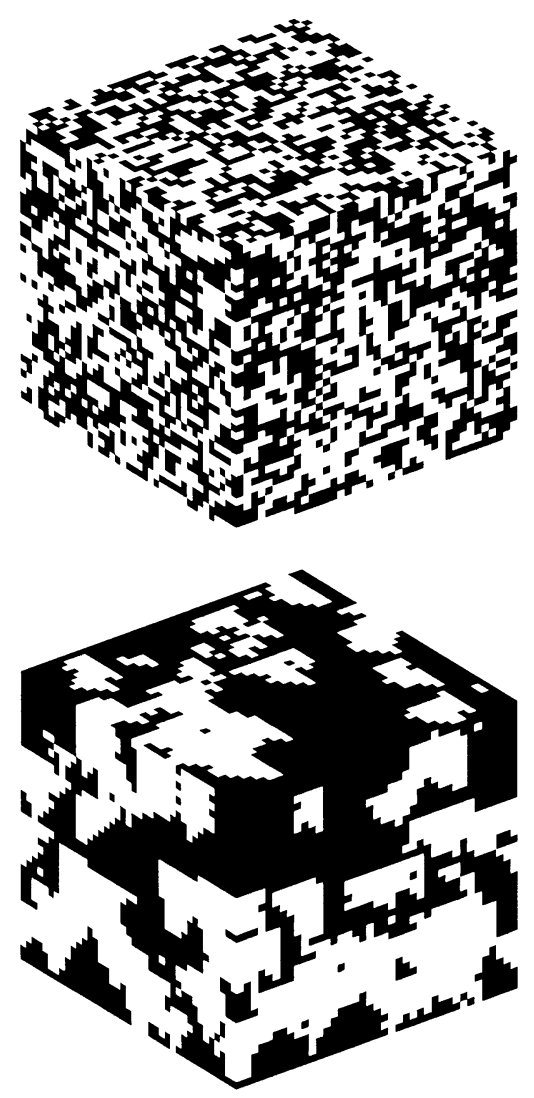

Figure 5. Spatial distribution of the local persistence at time $t^{*}$ such that $P\left(t^{*}\right)=1 / 2$ (i.e., 50 of sites, shown in black, have flipped by time $t^{*}$ ) at temperatures $T=1.0$ (top) and $T=0.15$ (bottom) for a system size $L=40$ in both cases. The appearance of dynamic critical fluctuations when $T \rightarrow 0$ is evident.

in the present coarse-grained model. We show in Figure 6 the analogue of spatial clustering of subsets of fast and slow sites. To build these snapshots, we represent a given percentage, $5 \%$, of the sites that relax faster or slower; that is, we show those sites for which the persistence time is among the 5\% smaller or larger in a randomly chosen run at temperature $T=0.18$.

One observes that the fastest sites are not randomly located in space, but clustered in "noncompact" or "stringy" objects, similar to those observed in simulations and experiments. ${ }^{36-38}$ The shape of these objects is a consequence of the existence of point defects of mobility. When a defect moves, it induces those sites along its trajectory to relax, so leaving in its wake a string of fast sites.

In Figure 6, we also show 5\% of the sites which are slowest, using the same simulation temperature as before. A more compact structure is seen. This is again the consequence of the relaxation via point defects of mobility. The slowest sites belong to regions of space devoid of defects which take then a very long time to be visited by defects. These large domains are thus slowly relaxed. It is the bulk of these slow domains that is observed in Figure 6. Note finally that, at large times, the distribution of slow cluster sizes seems very wide, since some isolated sites which have been not been visited by defects coexist with the very large domains discussed above.

Since our comments on Figure 6 are mainly qualitative, it should come as no surprise that snapshots built in this fashion in the isotropic case are very similar. ${ }^{14}$

Finally, it is interesting to compare these figures to those in previous publications, ${ }^{6,21}$ in which space-time diagrams of onedimensional kinetically constrained models were presented. There, spatio-temporal "bubbles" of immobile regions, bounded 

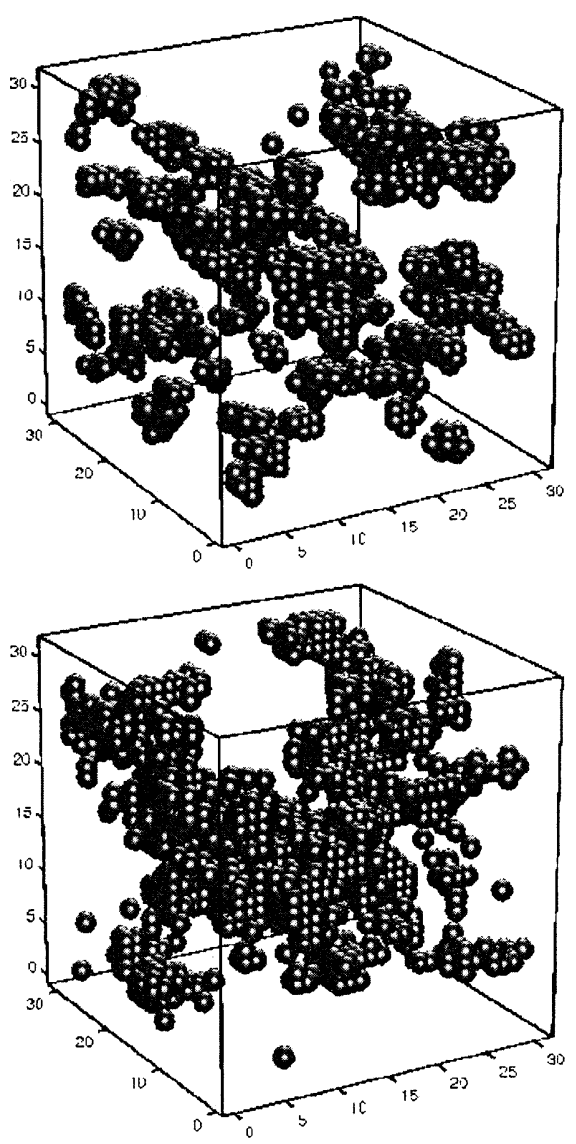

Figure 6. Visualization of $5 \%$ of the sites that relax faster (top) and slower (bottom) in a given run at $T=0.18$ for a system size $L=30$. Fast/slow sites are painted as spheres to highlight the similarity between heterogeneity in lattice models to that in atomistic models. The fast clusters in the top figure are less compact than the slow ones in the bottom figure as a consequence of relaxation via point like excitations. See the text for details.

by diffusing point defects, were presented. In the present model, these bubbles become $(3+1)$-dimensional objects, and Figure 6 shows their three-dimensional spatial projections of trajectories of various time extensions.

B. Spatial Correlations. We now quantify these qualitative observations via appropriate statistical correlators. We measure spatial correlations of the local dynamics via the following spatial correlator of the local persistence function:

$$
C(r, t, T)=\frac{1}{N f(t)} \sum_{i=1}^{N}\left[\left\langle P_{i}(t) P_{i+r}(t)\right\rangle-P^{2}(t)\right]
$$

where the function $f(t)=P(t)-P^{2}(t)$ in the denominator ensures the normalization $C(r=0, t, T)=1$. Alternatively, one can take the Fourier transform of (12), giving the corresponding structure factor of the dynamic heterogeneity

$$
S(q, t, T)=\frac{1}{N f(t)} \sum_{k, l=1}^{N}\left[\left\langle P_{k}(t) P_{1}(t)\right\rangle-P^{2}(t)\right] \mathrm{e}^{i q(k-1)}
$$

for wavevectors defined in the first Brillouin zone of the cubic lattice.

Finally, the zero wavevector limit of $S(q, t, T)$ defines a dynamic "four-point" susceptibility, $\chi(t, T)=S(q=0, t, T)$, which can be rewritten as the normalized variance of the (unaveraged) persistence function, $p(t) \equiv N^{-1} \sum_{i=1}^{N} P_{i}(t)$

$$
\chi(t, T)=\frac{N}{f(t)}\left[\left\langle p^{2}(t)\right\rangle-\langle p(t)\rangle^{2}\right]
$$

It should be noted that normalizations also ensure that $\chi(t, T)$ remains finite in the thermodynamic limit, except at a dynamic critical point such as the ones discussed in refs 13 and 39 .

Figure 7 shows the time dependence of the susceptibility (13) for various temperatures. The behavior of $\chi$ is similar to that observed in atomistic simulations of supercooled liquids. ${ }^{9}$ The susceptibility develops, at low temperature, a peak whose amplitude increases and whose position shifts to larger times as $T$ decreases. As expected, the location of the peak scales with the relaxation time $\tau(T)$, indicating that dynamical trajectories are maximally heterogeneous when $t \approx \tau(T)$.

In Figure 8, we show the correlator $C(r, t, T)$ and the structure factor $S(q, t, T)$ for different temperatures and fixed times $t=$ $\tau(T)$ where dynamic heterogeneity is maximal. These correlation functions confirm, as suggested by Figure 5, that a dynamic length scale associated with spatial correlations of mobility develops and grows as $T$ decreases: spatial correlations decay more slowly with distance as $T$ decreases, whereas a peak in the structure develops and grows at $q=0$.

It is possible to extract numerically the value of the corresponding dynamic length scale, $\xi(T)$, at each temperature. To do so, we study in detail the shape of the correlation functions shown in Figure 8. As for standard critical phenomena, we find that the dynamic structure factor can be rescaled according to

$$
S(q, \tau, T) \sim \chi(\tau, T) S(q \xi)
$$

where the scaling function $\int(x)$ has the following asymptotic behaviors in the scaling regime, $\xi \gg 1$ :

$$
\begin{aligned}
& \int(x \rightarrow 0) \sim \text { const } \\
& \int(x \rightarrow \infty) \sim x^{2-\eta}
\end{aligned}
$$

Both the susceptibility $\chi$ and the dynamic length scale $\xi$ estimated at time $t=\tau$ behave as power laws of the defect concentration

$$
\chi \sim c^{-\gamma}, \quad \xi \sim c^{-v}
$$

These relations imply that the exponents $\gamma$ and $v$ should be numerically accessible by adjusting their values so that a plot of $c^{\gamma} \int$ versus $q c^{-v}$ is independent of temperature. We show such a plot in Figure 9, and we find that the values $\gamma \approx 1$ and $v \approx$ $1 / 3$ lead to a good collapse of the data. The exponent $\gamma$ can be independently and more directly estimated from Figure 7 by measuring the height of the maximum of the susceptibility for various concentrations. The result is also well fitted to the power law $c^{\gamma}$ with $\gamma \approx 1$. We find also that the scaling function $S(x)$ is well-described by an empirical form $S(x)=1 /\left(1+x^{2-\eta}\right)$, consistent with eq 16 . Thus, we can determine the value of the "anomalous" exponent, $\eta$. We find that the theoretically expected $\eta=-\ln 3 / \ln 2 \approx-1.58$ describes the data very well. ${ }^{6}$ The fact that this exponent is much more negative than in the strong case of the FA model quantifies our observation that domains of Figure 5 were much less rough in the NEF model than in the FA model; that is, that dynamic domains of fragile systems have smoother boundaries than those of strong ones.

The spatial correlator $C(r, \tau, T)$ also obeys scale invariance, and we find that

$$
C(r, \tau, T) \sim \mathcal{O}\left(\frac{r}{\xi}, \tau\right)
$$




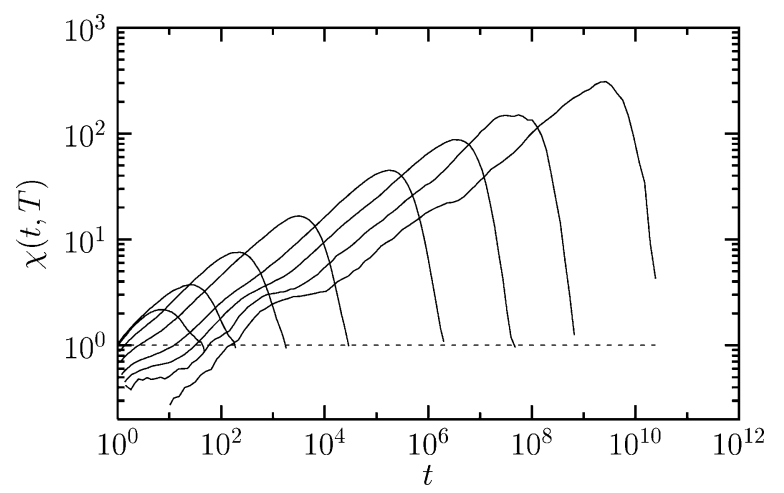

Figure 7. Dynamic 'four-point' susceptibility, eq 13, at temperatures $T=1.0,0.6,0.4,0.3,0.23,0.2,0.18$, and 0.16 (from left to right).
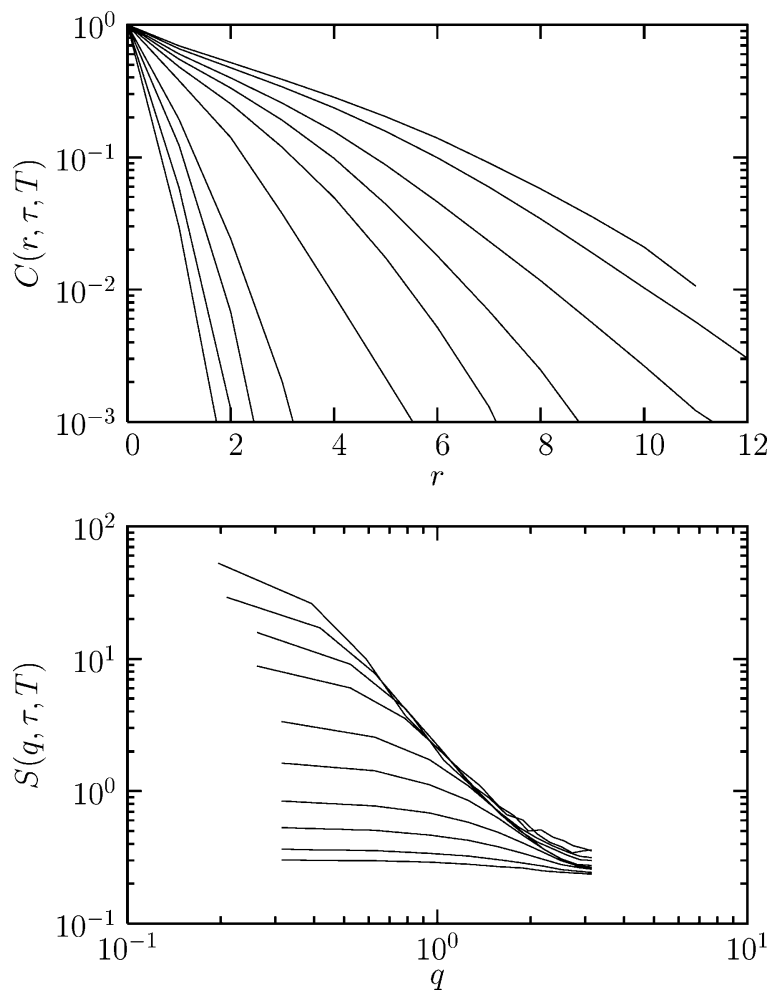

Figure 8. (Top) Spatial correlator of dynamic heterogeneity at the relaxation time $\tau(T)$. Temperatures as in Figure 7 decrease from left to right. (Bottom) Corresponding structure factor. Temperatures as in Figure 7 decrease from bottom to top.

with the asymptotic behavior $\mathscr{G}(x \rightarrow 0) \sim x^{-0.58}$, as a result of a generalized Porod's law. The scaling behavior (18) is presented in Figure 9, which also confirms the temperature dependence of the correlation length, $\xi \sim c^{-1 / 3} \cdot{ }^{12}$ It is interesting to note that the relation between susceptibility and length is very different in the strong and fragile cases, since we find that $\chi \sim$ $\xi^{3}$ in the NEF model, whereas the scaling is closer to $\chi \sim \xi^{2}$ in the FA case. ${ }^{14}$

\section{Comparison to Experimental Data}

In this section, we use the numerical results obtained in previous sections to fit experimental data with the NEF model. In doing so, there are several points that need to be considered.

First, the model we use is meant to be a description of supercooled liquids which is coarse-grained both in time and space and lives on a lattice. We are thus dealing with discrete, rather than continuous, spatial degrees of freedom, and the very short-time dynamics of the liquid is removed. By construction,
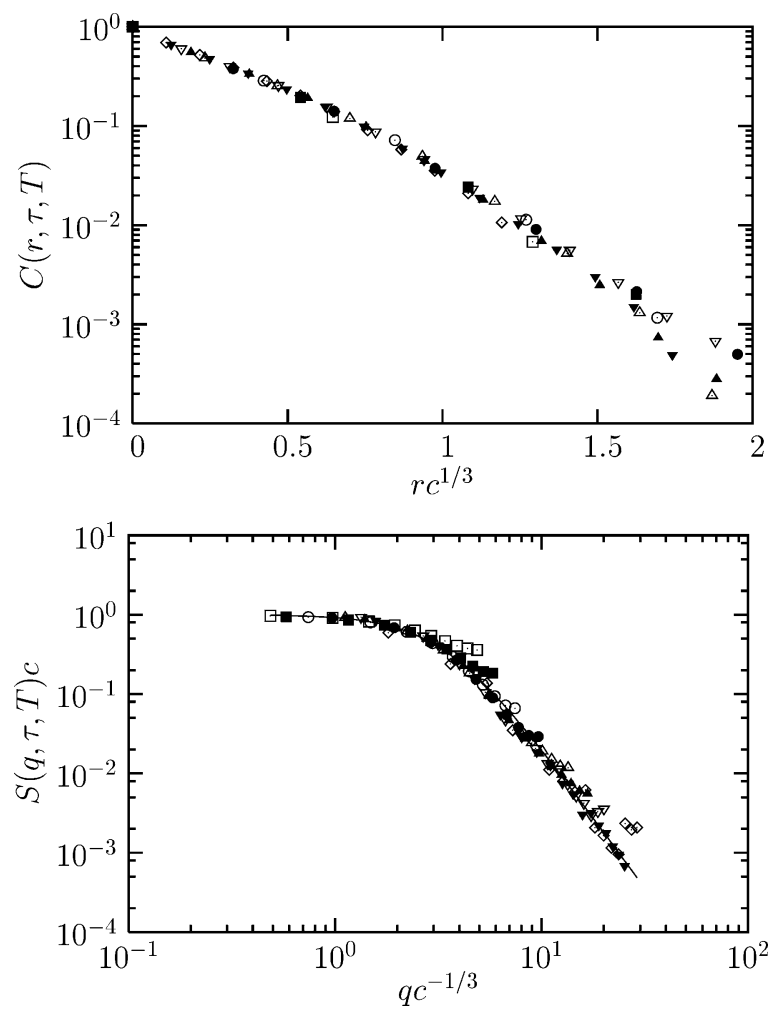

Figure 9. (Top) Spatial correlator rescaled with the form $C(r) \sim G(r)$ $\xi), \xi \sim c^{-1 / 3}$. (Bottom) Rescaled structure factor with $\chi=S(q=0) \sim$ $c^{-1}$ and $\xi \sim c^{-1 / 3}$. Full line interpolates between $S(q \rightarrow 0) \sim$ const and $S(q \rightarrow \infty) \sim k^{-3.58}$.

this produces discrepancies between real data on liquids and numerical NEF model data, especially for short times and small lengths. This is a small price to pay in such an approach given the large number of features that can still be satisfactorily accounted for with the NEF model.

Second, we have some freedom on how to relate real experimental time scales and Monte Carlo steps in the simulation. This will be done empirically, and we find that the expected equivalence, $1 \mathrm{MC}$ step $\approx$ few $\mathrm{ps},{ }^{12}$ works well, independently of the temperature. Explicitly, we found that $1 \mathrm{MC} \in[1,10] \mathrm{ps}$ for the whole range of experimental data we have considered. . $^{31,41}$

Third, one must adjust the temperature given in Kelvin in experiments to the adimensional $T$ of the simulations. This amounts to fitting the value of an energy scale, $J$, which should appear on dimensional grounds in front of the Hamiltonian (1). In principle, $J$ could be fixed independently by fitting, say, viscosity data of a given liquid before using the corresponding temperatures to fit more detailed dynamic data in what would be a zero-parameter fitting procedure. We have done the correspondence in a less constrained manner, adjusting $T$ in the simulation to give a good fit to the data. Very satisfactorily, though, we end up with a correspondence between numerical and experimental temperatures which is well described by linear relations, as it should be at low temperatures. ${ }^{12}$

In Figure 10, we show the result of this fitting procedure as applied to recent data measured using the so-called optical Kerr effect. ${ }^{40,41}$ This technique has several advantages. It extends for over 5 orders of magnitude in time. The quantity measured is the derivative of a time correlation function and, therefore, the analogue of the distribution of time scales, $\pi(t)$, discussed above. Also vibrations, which are neglected in our approach, affect very little the measured decay in the time scales of interest. 


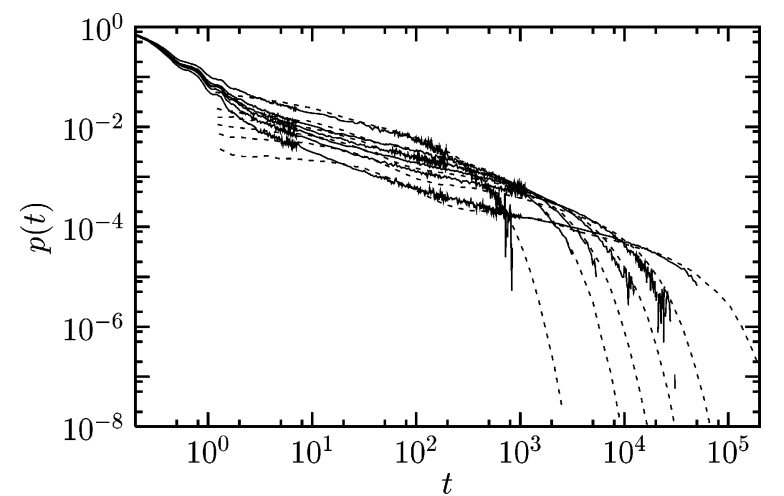

Figure 10. Optical Kerr effect experimental for supercooled liquid 2-biphenylmethanol (full lines from ref 41) at temperatures $T=359$, $327,319,311,303$, and $291 \mathrm{~K}$ (from left to right). Dashed lines are corresponding data for the NEF model at temperatures $T=0.4,0.34$, $0.32,0.3,0.28$, and 0.25 (from left to right). Time is counted in picoseconds in the experiments, in Monte Carlo steps in simulations.

In ref 41 , the experimental results were fitted using the empirical form

$$
\pi(t)=\left[p t^{-1+C}+d t^{b-1}\right] \exp (-t / \tau)
$$

with the values $b \approx 0.8-0.85$ and $C \approx 0 .-0.2$ consistently found in different liquids. The $1 / t$ behavior of $\pi(t)$ obtained at short times when $C=0$ implies a $\log t$ behavior of the time correlator. This was taken as a challenging result to modecoupling theory which does not naturally predict such patterns, as can be seen when asymptotic analytic results are considered. ${ }^{34}$ These data were however recently virtually perfectly fitted using a standard schematic version of the mode-coupling theory, therefore nullifying the criticisms raised in the experimental work. ${ }^{35}$ Note that several fitting parameters are allowed by the mode-coupling fits, which are performed using a two-correlator schematic model: coupling constant, distance to the dynamic singularity, and various numerical factors adapting theoretical time scales to the experimental ones. By contrast, we make use of just one free parameter in Figure 10.

The "nearly logarithmic decay" of correlations has a simple explanation in our approach. At modest temperatures, $T \lesssim T_{0}$, there is a coexistence of isolated excitations, responsible for "slow processes", and rapidly relaxing clusters of excitations; see ref 21 for a detailed discussion of the related temperature crossovers. This coexistence manifests as a nearly flat distribution $\pi(\log t)$ over a wide range of time scales. This translates into a $1 / t$ behavior of $\pi(t)$ and logarithmic decay of $P(t)$, as observed in Figure 10. This explanation, moreover, predicts that the effective exponent $C$ appearing in eq 19 should acquire a slight temperature dependence and change from $C<0$, when fast processes dominate closer to $T_{0}$, to $C>0$ at lower temperature when slow processes become dominant. This is precisely what is found in experiments. ${ }^{41}$ This subtlety is also accounted for by mode-coupling theory. ${ }^{35}$

The only real discrepancy between fits and data is visible at very short time, as was anticipated, but the overall quantitative agreement is very good. As already suggested by the qualitative analysis of ref 21 , our theoretical approach can be used even far above the experimental glass transition. Mode-coupling theory is thought to be applicable in this regime, under the assumption that the dynamics at modest supercooling is different from that near $T_{\mathrm{g}}$. As in ref 21, our results here suggest that this may not be the case.

One of the advantages of the present approach on modecoupling theory is that it does not produce an unphysical

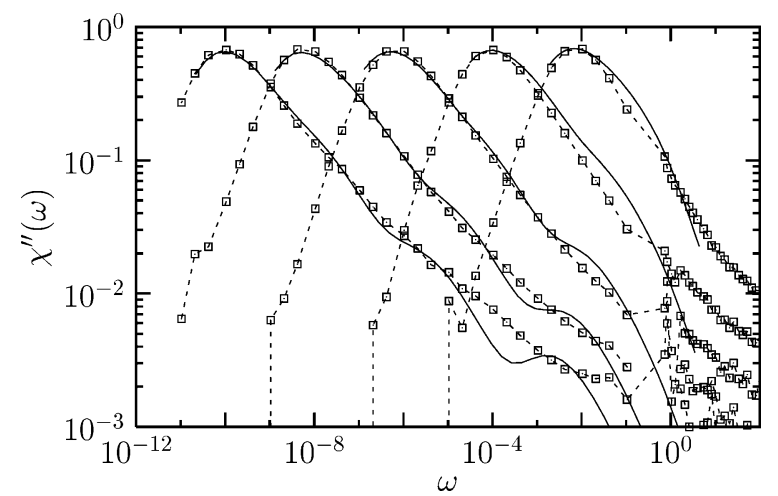

Figure 11. Dielectric susceptibility data for Salol obtained from the authors of ref 42 at temperatures $T=255,243,233,225$, and $219 \mathrm{~K}$ (from right to left). Full lines are corresponding data for the NEF model at temperatures $T=0.385,0.26,0.198,0.168$, and 0.15 (from left to right). Time is counted in units of $10 \mathrm{ps}$ in the experiments, and in Monte Carlo units in the simulations.

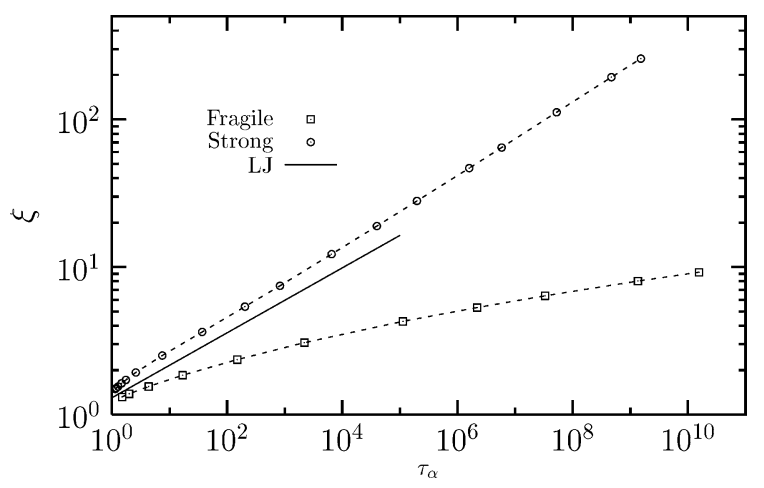

Figure 12. Dynamic scaling of time scales versus lengthscales in the strong three-dimensional FA model, ${ }^{13}$ the fragile three-dimensional NEF model, and three-dimensional binary Lennard-Jones mixture. ${ }^{13}$

singularity at a temperature above $T_{\mathrm{g}}$, and it can therefore be used down to very low temperatures and large relaxation times. In Figure 11, we use the NEF model to fit dielectric data taken on Salol. ${ }^{31}$ As before, the fits are done using a single free parameter. Figure 11 shows that the overall agreement is again very good. Note in particular that the high-frequency wing is correctly accounted for by the (discrete) hierarchy of dynamic lengthscales discussed in the previous sections.

Again, discrepancies due to coarse-graining are evident: absence of short-time processes and discreteness of the hierarchy of time scales. Discrete scales are particularly evident in the Nagel wing, where numerical data only produce the "skeleton" of the wing instead of a smooth curve. Note that the same experimental data were fitted in ref 42 using a frustration limited domain scaling picture of the glass transition: seven fitting parameters were used there to obtain a satisfactory fit. Such data are usually fitted in experimental papers by empirical forms involving again several free parameters. ${ }^{30}$

Consider, finally, the scaling of lengths and times. Figure 12 shows dynamic scaling in three different model systems: the three-dimensional FA model (indicated as "strong"; data from ref 14), the present NEF model data (indicated as "fragile"), and the Kob-Andersen Lennard-Jones binary mixture ${ }^{43}$ ("LJ" in the figure; data from ref 13). The figure shows that in the fragile case the growth of dynamic lengthscales is much less pronounced than in the strong one. This is one of the central predictions of the dynamic facilitation approach. ${ }^{12}$ It is a consequence of the temperature dependent dynamic exponent of east-like models such as the NEF model, in contrast to strong systems where dynamic lengths go as a fixed power of the 
relaxation time. Note that a figure qualitatively similar to Figure 12 would be obtained for the four-point susceptibility as a function of relaxation time.

This slow growth of lengthscales in the NEF model is also compatible with the modest size of heterogeneities as suggested by experiments near $T_{\mathrm{g}}{ }^{8}$ Interestingly, the LJ mixture appears in this representation as a fairly strong system, despite the common assumption that it is a good model for fragile liquids. The theoretical and numerical findings reported in Figure 12 offer a solution to the paradox that experimentally measured length scales are very small when compared to extrapolations performed from numerical works. ${ }^{8,9}$

\section{Conclusions}

In summary, we have presented an extensive numerical study of the NEF model, the three-dimensional generalization of the east facilitated spin model. The NEF model, whose dynamical rules have an externally imposed asymmetry, is in the same universality class of the more physical arrow model of ref 12 in the limit of maximal directional persistence and therefore models the behavior of fragile, super-Arrhenius, glass formers.

We have characterized the equilibrium dynamics of the NEF model through measurements of the $\alpha$-relaxation time scales, distributions of relaxation times, and the growth of dynamic correlation lengths and four-point susceptibilities. We find that $\tau_{\alpha}$ follows a Bässler law of exponential-inverse-squared temperature dependence and that the dynamic correlation length grows as $\xi \sim c^{-v}$ with $v \approx 1 / 3$, where $c$ is the equilibrium concentration of excitations, whereas the dynamic susceptibility grows as $\chi \sim c^{-\gamma}$ with $\gamma \approx 1$. These results confirm some of the expectations of ref 12 for the arrow model, in particular the quasi one-dimensional nature of the dynamics in all dimensions. The decay of the four-point structure factor, $S(k) \approx k^{-2-1.58}$, is a direct generalization of the result for the 1D east model, ${ }^{6}$ and an indication of hierarchical dynamics in the NEF model. These two features, persistence of directionality and hierarchical dynamics, give rise to fragile behavior. ${ }^{12}$

We have shown how the NEF model can be used to rationalize experimental observations over a wide range of temperatures and time scales. We have fitted correlation data in the time domain, obtained in the mildly supercooled regime by optical Kerr effect, ${ }^{41}$ with the NEF model predictions. This provides an interpretation of the quasilogarithmic time dependence of correlations based on the coexistence of isolated excitations and clusters of excitations, which occurs when the system is crossing over from a regime of homogeneous dynamics at high $T$ to one of heterogeneous dynamics at low $T .^{21}$ We have shown how the NEF model can also be used to describe dielectric susceptibility data, in the frequency representation, measured close to the glass transition by dielectric spectroscopy. Interestingly, the NEF model successfully accounts for the excess high-frequency or Nagel wing. ${ }^{31}$

The results presented in this paper add to the list $\mathrm{t}^{12,19,29,44}$ of phenomenological observations that can be quantitatively understood within the dynamic facilitation approach.

Acknowledgment. We are grateful to the authors of refs 30,41 , and 42 for providing us with their published data and to the authors of ref 35 for sharing their published theoretical results. We thank G. Biroli, J.-P. Bouchaud, W. Götze, G. Tarjus, and especially D. Chandler for stimulating discussions and comments. This work was supported by CNRS (France), EPSRC Grant Nos. GR/R83712/01 and GR/S54074/01, and University of Nottingham Grant No. FEF 3024.

\section{References and Notes}

(1) Ritort, F.; Sollich, P. Adv. Phys. 2003, 52, 219.

(2) Fredrickson, G. H.; Andersen, H. C. Phys. Rev. Lett. 1984, 53, 1244; J. Chem. Phys. 1985, 83, 5822.

(3) Jäckle, J.; Eisinger, S. Z. Phys. B 1991, 84, 115

(4) Sollich, P.; Evans, M. R. Phys. Rev. Lett. 1999, 83, 3238.

(5) Harrowell, P. Phys. Rev. E 1993, 48, 4359.

(6) Garrahan, J. P.; Chandler, D. Phys. Rev. Lett. 2002, 89, 035704

(7) Sillescu, H. J. Non-Cryst. Solids 1999, 243, 81.

(8) Ediger, M. D. Аnпи. Rev. Phys. Chem. 2000, 51, 99

(9) Glotzer, S. C. J. Non-Cryst. Solids 2000, 274, 342.

(10) Richert, R. J. Phys: Condens. Matter 2002, 14, R703.

(11) Other generalizations of the east model include the north-and-east model, where a site can only change its state if two of its neighbours, for example to the east and north, are simultaneously in their excited state; see: Reiter, J.; Mauch, F.; Jackle, J. Physica A 1992, 184, 493.

(12) Garrahan, J. P.; Chandler, D. Proc. Natl. Acad. Sci. U.S.A. 2003, 100,9710

(13) Whitelam, S.; Berthier, L.; Garrahan, J. P. Phys. Rev. Lett. 2004, 92, 185705 .

(14) Whitelam, S.; Berthier, L.; Garrahan, J. P. cond-mat/0408694.

(15) Beyond the one-spin facilitated FA model, Fredrickson and Andersen have also introduced more constrained models, namely $n$-spin isotropically facilitated models with $n>1$. These models do exhibit fragile behaviour, ${ }^{2}$ but they are in a sense not realistic since they are characterized by exponentially diverging activation energies, ${ }^{16}$ a feature not observed in real liquids.

(16) Toninelli, C.; Biroli, G.; Fisher, D. S. Phys. Rev. Lett. 2004, 92, 185504 .

(17) Newman, M. E. J.: Barkema, G. T. Monte Carlo Methods in Statistical Physics; Oxford University Press: Oxford, U.K., 1999.

(18) Berthier, L. Phys. Rev. Lett. 2003, 91, 055701.

(19) Berthier, L.; Chandler, D.; Garrahan, J. P. Europhys. Lett. 2005, 69,230 .

(20) For a detailed study of correlation in the east model, especially the behaviour of the persistence at very long times (beyond the alpha-relaxation time regime of interest to us here), see: Sollich, P.; Evans, M. R. Phys. Rev. E 2003, 68, 031504.

(21) Berthier, L.; Garrahan, J. P. Phys. Rev. E 2003, 68, 041201.

(22) Brumer, Y.; Reichman, D. R. Phys. Rev. E 2004, 69, 041202.

(23) Eisinger, S.; Jackle, J. J. Stat. Phys. 1993, 73, 643.

(24) Aldous, D.; Diaconis, P. J. Stat. Phys. 2002, 107, 945

(25) Bässler, H. Phys. Rev. Lett. 1987, 58, 767.

(26) Adams, G.; Gibbs, J. H. J. Chem. Phys. 1958, 43, 139.

(27) Richert, R.; Angell, A. J. Chem. Phys. 1998, 108, 9016.

(28) Buhot, A.; Garrahan, J. P. Phys. Rev. E 2001, 64, 21505.

(29) Berthier, L.; Garrahan, J. P. J. Chem. Phys. 2003, 119, 4367.

(30) Blochowicz, T. Tschirwitz, C.; Benkhof, S.; Rössler, E. A. J. Chem. Phys. 2003, 118, 7544.

(31) Dixon, P. K.; Wu, L.; Nagel, S. R.; Williams, B. D.; Carini, J. P. Phys. Rev. Lett. 1990, 65, 1108. Schneider, U.; Brand, R.; Lunkenheimer, P.; Loidl, A. Phys. Rev. Lett. 2000, 84, 5560.

(32) Brodin, A.; Bergman, R.; Mattsson, J.; Rössler, E. A. Eur. Phys. J. B 2003, 36, 349 .

(33) Leheny, R. L. Phys. Rev. B 1998, 57, 10537

(34) Götze, W. J. Phys.: Condens. Matter 1999, 11, A1.

(35) Götze, W.; Sperl, M. Phys. Rev. Lett. 2004, 92, 105701.

(36) Donati, C.; Douglas, J. F.; Kob, W.; Plimpton, S. J.; Poole, P. H.; Glotzer, S. C. Phys. Rev. Lett. 1998, 80, 2338

(37) Hurley, M. M.; Harrowell, P. Phys. Rev. E 1995, 52, 1694

(38) Weeks, E. R.; Crocker, J. C.; Levitt, A. C.; Schofield, A.; Weitz, D. A. Science 2000, 287, 627.

(39) Kirkpatrick, T. R.; Thirumalai, D. Phys. Rev. A 1988, 37, 4439 Franz, S.; Parisi, G. J. Phys.: Condens. Matter 2000, 12, 6335. Biroli, G.; Bouchaud, J.-P. Europhys. Lett. 2004, 67, 21.

(40) Torre, R.; Bartolini, P.; Pick, R. M. Phys. Rev. E 1998, 57, 1912

(41) Hinze, G.; Brace, D. D.; Gottke, S. D.; Fayer, M. D. Phys. Rev Lett. 2000, 84, 2437; J. Chem. Phys. 2000, 113, 3723. Cang, H.; Novikov, V. N.; Fayer, M. D. Phys. Rev. Lett. 2003, 90, 197 401; J. Chem. Phys. 2003, 118, 2800 .

(42) Viot, P.; Tarjus, G.; Kivelson, D. J. Chem. Phys. 2000, 112, 10368.

(43) Kob, W.; Andersen, H. C. Phys. Rev. Lett. 1994, 73, 1376.

(44) Jung, Y. J.; Garrahan, J. P.; Chandler, D. Phys. Rev. E 2004, 69, 061205 . 